\title{
Voltaire y Condorcet: Del Essai al Esquisse*
}

\author{
Adrián Ratto ${ }^{1}$ \\ Universidad de Buenos Aires (Argentina) \\ Consejo Nacional de Investigaciones Científicas y Técnicas (CONICET)
}

Recibido: septiembre 21 de 2015 - Revisado: octubre 27 de 2015 - Aceptado: noviembre 8 de 2015

Referencia formato APA: Ratto, A. (2015). Voltaire y Condorcet: Del Essai al Esquisse. Revista Cientifica Guillermo de Ockham, 13(2), 17-23.

\section{Resumen}

En 1770, Condorcet conoció a Voltaire en su residencia de Ferney. Desde ese momento mantuvieron un estrecho contacto. En 1789, escribió una Vie de Voltaire, en la que reconoce su deuda con la filosofía de la historia del autor de la Henriade. El propósito de este trabajo es demostrar, a partir del examen analítico de las fuentes y otros documentos, que existen, sin embargo, tensiones entre los trabajos históricos e historiográficos de los filósofos y que en ciertos aspectos el Esquisse d'un tableau historique des progrès de l'esprit humain de Condorcet es un paso atrás con respecto al Essai sur les mours, de Voltaire. Con el fin de alcanzar ese objetivo, se reconstruye someramente el programa historiográfico que Voltaire elabora en los años cuarenta durante el período de Cirey, luego se analiza su célebre Essai, para observar la aplicación del método, y finalmente se evalúa el Esquisse a la luz del proyecto volteriano, del que Condorcet se declara deudor.

Palabras clave: Condorcet, Voltaire, historia, método, espíritu humano

\section{Voltaire and Condorcet: From the Essai to the Esquisse}

\section{Abstract}

In 1770, Condorcet met Voltaire at Ferney. From that moment they kept in close contact. In 1789, he wrote Vie de Voltaire, where he acknowledges his debt with the philosophy of the history of the author of the Henriade. The aim of this paper is to show, from the analytical examination of different sources, that there are, nevertheless, some tensions between historical and historiographic works of the philosophers and that to a certain extent Condorcet's Esquisse d'un tableau historique des progrès de l'esprit humain is a step backwards from Voltaire's Essai sur les mœurs. In order to reach that objective, we briefly rebuild the historiographic programme that Voltaire developed during Cirey's 40's, then his famous Essai is analysed in order to observe the application of the method and finally we study the Esquisse in the light of the Voltairean project, to which Condorcet declares himself indebted.

Keywords: Condorcet, Voltaire, history, method, human spirit

\footnotetext{
* Este trabajo fue realizado en el marco de los proyectos Worldbridges: Philosophy of History and Globalisation of Knowledge. Cultural Bridges Between Europe and Latin America [Marie Curie International Research Staff Exchange Scheme (F7-PEOPLE-2013-IRSES: 612644)] y PICT-2013-0660: Del tiempo de la experiencia a la experiencia del tiempo histórico [Agencia Nacional de Promoción Científica y Tecnológica, Ministerio de Ciencia, Tecnología e Innovación Productiva, Argentina].

1. Doctor en Filosofía por la Universidad de Buenos Aires. Actualmente se desempeńa como becario postdoctoral del Consejo Nacional de Investigaciones Científicas y Técnicas (Conicet) y como docente en la Facultad de Filosofía y Letras de la Universidad de Buenos Aires. Dirección postal: Av. Luis María Campos 381, 3 “A”, Ciudad Autónoma de Buenos Aires, Argentina. CP: 1426. E-mail: ga_ratto@yahoo.com.ar
} 


\section{Voltaire e Condorcet: Do Essai ao Esquisse}

\section{Resumo}

Em 1770, Condorcet conheceu Voltaire na sua residência de Ferney. A partir desse momento, mantiveram um contato estreito. Em 1789, escreveu uma Vie de Voltaire, na qual reconhece a sua dívida com a filosofia da história do autor da Henriade. O propósito desse trabalho é o de demonstrar, a partir do exame analítico das fontes e outros documentos, que existem, porém, tensões entre os trabalhos históricos e historiográficos dos filósofos e que, em alguns aspectos, o Esquisse d'un tableau historique des progrès de l'esprit humain de Condorcet representa um passo atrás respeito do Essai sur les mœurs de Voltaire. Com a finalidade de alcançar tal objetivo, reconstrói-se sumariamente o programa historiográfico que Voltaire elabora nos anos '40, durante o período de Cirey; posteriormente, analisa-se o seu célebre Essai, para assim observar a aplicação do método, e finalmente avalia-se o Esquisse sob a luz do projeto volteriano, do qual Condorcet se declara em dívida.

Palavras-chave: Condorcet, Voltaire, história, método, espírito humano

\section{Introducción}

El 16 de septiembre de 1770 Nicolas de Caritat, marqués de Condorcet, partió junto a Jean le Rond d'Alembert hacia Italia. ${ }^{2}$ Siete días más tarde, el día 23 de ese mismo mes, los viajeros se detuvieron en la residencia de Voltaire en Ferney, donde permanecieron dos semanas (Voltaire y d'Alembert se conocían desde 1756 y se escribían regularmente). Allí, el joven Condorcet (tenía en ese momento veintiséis años) conoció a Voltaire, con quien estableció un estrecho contacto. Los dos filósofos mantuvieron hasta 1778 -año de la muerte de Voltaireuna fluida correspondencia y lucharon juntos en 1774 por la revisión del proceso del caballero de la Barre, un caso de intolerancia religiosa. ${ }^{3}$ En una carta del 9 de abril de 1777, Voltaire escribía a Condorcet: "quisiera que me prometáis para consolarme, que vais a tomar mi lugar en la academia de las palabras" (Voltaire, 1882, p. 215). Tras el deceso de Voltaire, Condorcet se encargó de editar sus obras completas ${ }^{4}$ y escribió en 1789 una Vie de Voltaire, en la que reconoce su deuda con el filósofo, particularmente en el terreno de la teoría de la historia.

El objetivo de este trabajo es demostrar que el intento de Condorcet de aplicar el método historiográfico de Voltaire en su Esquisse d'un tableau historique des progrès de l'esprit humain (1795, publicación póstuma) fracasa en la medida en que el autor no cumple en ese trabajo con los requisitos que, según Voltaire, debe cumplir la historia moderna.

La estructura del trabajo es como sigue. En primer lugar, se reconstruirá someramente el proyecto historiográfico que Voltaire presenta en diferentes escritos en los años cuarenta; luego, se examinará la aplicación que el filósofo francés hace del método en su Essai sur les mours, y finalmente, se demostrará que Condorcet no sigue en el Esquisse los lineamientos del método volteriano, a pesar de reconocer su deuda con la epistemología de la historia del autor de la Henriade.

\section{El proyecto historiográfico de Voltaire en los años cuarenta}

En 1742 Voltaire publicó el trabajo Remarques sur l'histoire y dos años después, en 1744, Nouvelles considerations sur l'histoire. En estos textos presenta un programa para la renovación de la historia, el cual formaba parte de su proyecto de introducir los trabajos de Isaac Newton en Francia. Con ese fin había escrito los Eléments de la philosophie de Newton (1738), mientras la marquesa Émilie du Châtelet, junto a quien vivía en Cirey, traducía al francés la Philosophie Naturalis Principia Mathematica, del físico inglés. ${ }^{5}$ El trabajo de Newton había sido publicado en Londres en 1687, pero los franceses eran aún devotos

2. A propósito de los detalles de ese viaje, véase Chouillet, 1996; Chouillet y Crépel, 1994a.

3. Acerca de la relación entre Condorcet y Voltaire, véase Chouillet, 1994b; Michael, 1987.

4. Esa edición de las obras de Voltaire es llamada por los especialistas edición Kehl (Euvres complètes de Voltaire, edición de Jean-Antoine-Nicolas de Caritat de Condorcet (marqués de) y Pierre Augustin Caron de Beaumarchais. Kehl: Société littéraire-typographique, 1784-1789, 70 vols.). Los libros que componen la edición fueron parte del cortejo que en 1791 acompañó los restos de Voltaire hacia el panteón de los hombres ilustres de la patria (París).

5. La marquesa Émilie du Châtelet comenzó en 1736 la traducción de la obra, publicada originalmente en latín. La traducción del libro fue publicada en 1759, luego de la muerte de la marquesa. Acerca del interés por la ciencia de Voltaire y Émilie en los ańos treinta y cuarenta, véase Pomeau, 2001.

$18<$ Universidad de San Buenaventura, Cali - Colombia 
de la física de los torbellinos de Descartes. El fin de Voltaire -quien había entrado en contacto con las obras de Newton, Locke y Bacon, entre otros, durante su exilio en Inglaterra- no era solo modificar la interpretación de sus compatriotas acerca del mundo físico, sino también producir una revolución desde el punto de vista de la metodología del conocimiento. En ese marco se inscribe su intención de acercar la historia a los métodos de la física experimental.

Quizá pronto ocurra en la manera de escribir historia, lo que ha sucedido en la física. Los nuevos descubrimientos han vuelto obsoletos los antiguos sistemas [...]. Se querrá conocer al género humano con el mismo rigor alcanzado en la filosofía natural (Voltaire, 1957, p. 46). ${ }^{6}$

Acercar la historia a la ciencia a significaba para Voltaire-como se desprende de los dos trabajos mencionadosdos cosas: 1. ajustar el relato histórico a los "hechos", dejando de lado "los oráculos" y "las fábulas antiguas"; 2. ampliar los límites del campo de investigación. Voltaire se propuso escribir historia de manera científica, apoyando el relato en hechos, testimonios y registros públicos y tomando distancia respecto de las historias romanesques y las fábulas. Esto lo llevó a alejarse de aquellos que, como Géraud de Cordemoy (1626-1684), habían afirmado que el historiador tenía que ocuparse de la belleza y la claridad del estilo, antes que de la veracidad de la información acerca del pasado (Cordemoy, 1691, pp. 68, 69); pero también de Claude Fleury y su Histoire ecclésiastique (1691) y de Charles Rollin y su Histoire romaine depuis la fondation de Rome jusqu’à la bataille d'Actium (1738), quienes confundían la historia y la religión o retomaban en su época absurdos relatos de la Antigüedad. ${ }^{7}$ Por otro lado, Voltaire entendió que para poner la historia a la altura de la nueva ciencia, era necesario escribir una historia general, "una historia de los hombres". Es decir, la "historia moderna" no podía reducirse, según el francés, a la historia de una nación privilegiada, como ocurría en los Discours sur l'histoire universelle (1681) de Jacques Bénigne Bossuet, o a la superficial narración de la vida de las cortes y los reyes (Voltaire, 1957, p. 48). Así, se interesó en sus trabajos históricos por la historia de disciplinas y lugares que hasta ese momento no habían logrado atraer en general el interés de los historiadores: la demografía, la agricultura, la economía, las costumbres, China, la India, etc.

\section{La materialización del proyecto historiográfico de Voltaire en el Essai}

"Muchas personas saben que el Essai sur l'histoire général des moeurs, fue emprendido hacia 1740 con el objetivo de reconciliar con la ciencia de la historia a una dama ilustre que poseía todas las otras", decía Voltaire en 1763 (Voltaire, 1963b, p. 900). La dama era la mencionada marquesa Émilie du Châtelet, quien interesada por la ciencia no veía en la historia más que una disciplina caprichosa y carente de rigor. El Essai es, precisamente, el intento de Voltaire de adosar la historia a la ciencia, para reconciliar a Émilie con esa disciplina.

Para alcanzar ese objetivo, Voltaire necesitaba aplicar al pasado el método historiográfico que había elaborado a la luz de sus trabajos sobre Newton. Por una parte, era necesario llevar a cabo un cuidadoso trabajo sobre las fuentes y testimonios, analizar los hechos y eliminar los adornos y las fantasías. Por otro, dejar de lado la historia de los reyes y los dioses y hacer una "historia del espíritu humano"; una "historia de los hombres", de sus costumbres, sus usos, sus artes y sus leyes (Voltaire, 1957, p. 44). Lo que merece ser conocido por esa dama ilustre, dice Voltaire en las primeras páginas del Essai, "es el espíritu, las costumbres, los usos de las principales naciones" (Voltaire, 1963a, p. 194). Trazar la historia de la "historia del espíritu humano", no es, para Voltaire, narrar la historia de un puñado de reyes y de las guerras que han provocado; menos aún, narrar la historia de una nación en particular o de un pueblo elegido, como había hecho Bossuet, quien, según Voltaire, creía que todo giraba en torno al pueblo judío (p. 196). Era necesario, para el pensador galo, descentralizar la historia.

En el marco de este proyecto, ${ }^{8}$ Voltaire se ocupa en el primer capítulo del Essai, de los usos, las leyes y las ciencias en China durante la Antigüedad. Reconoce sus tempranos avances en el campo de la astronomía y señala que en sus primeros libros no se encuentran prodigios o predicciones, lo que los hace, señala, más respetables. China, dice, era ya "una nación civilizada cuando nosotros no éramos más que salvajes" (p. 208). Asimismo, menciona sus conocimientos de geometría, sus invenciones, sus descubrimientos y la pureza de su religión, la cual no había sido desfigurada por las fábulas, ni había sido mezclada en guerras y disputas (p. 222).

6. Las traducciones de los trabajos de Voltaire y Condorcet son nuestras.

7. Para una visión de conjunto sobre la escritura de la historia en los siglos XVII y XVIII, véase Guion, 2008; Borghero, 1990.

8. Para una interpretación diferente, véase Méricam-Bourdet, 2012, p. 23. 
Luego de disertar sobre China, se refiere a la India. Reconoce que al igual que los chinos, los indios han cultivado las ciencias y las artes desde tiempos remotos (p. 228). Los griegos, apunta, habrían viajado hacia allí en busca de las ciencias y el célebre Pitágoras habría aprendido de los indios las propiedades del triángulo. Los indios -detalla Voltaire- trataron sobre filosofía y moral en forma de alegorías y parábolas y dieron forma a la más antigua teogonía. El Shasta es un libro indio que menciona a un ser eterno y a un conjunto de seres creados por este que participan de su esencia. Voltaire refiere que esto fue luego recogido, entre otros, por Platón y afirma que "nada hay en la antigüedad tan majestuoso ni tan filosófico" (p. 230). El filósofo concluye que la India "debe haber sido el lugar que se ha civilizado primero" y añade con admiración que la religión de los indios se reducía al "culto de un ser supremo" y estaba "desligada de toda superstición y de todo fanatismo" (p. 237). Igualmente, antes de formular sus opiniones sobre Grecia y Roma se refiere a Arabia. Señala que en esa época los árabes habían visto florecer las artes y las ciencias y pasado de la barbarie a la civilización (p. 267).

Voltaire se ocupó de respaldar sus afirmaciones por medio de testimonios directos o indirectos y documentos históricos. Así, utilizó la traducción de Desmaisons de los documentos de Abulcasis acerca de los tártaros (p. 609); para la historia del Vaticano, un Abrégé des Annales ecclésiastiques (1673), de César Baronius (p. 434), y sobre los judíos, los Voyages (1734), de Benjamin de Tudèle (p. 150). Su preocupación por el rigor de la información vertida en el libro lo llevó a introducir pasajes, precisar detalles y hacer modificaciones en las posteriores ediciones del Essai, ${ }^{9}$ lo cual no impidió que algunos -como el jesuita ClaudeFrançois Nonnotte, quien publicó en 1762 Les erreurs de Voltaire-cuestionaran al filósofo sus errores. Sin embargo, en muchos casos esos "errores" no estaban vinculados a los datos históricos, sino a asuntos religiosos. De todas maneras, por mucho que le dolieran las críticas, no dudó en servirse de ellas cuando las consideraba acertadas. Así, por ejemplo, corrigió en 1769 partes de su Philosophie de l'histoire (1765), que desde ese año formaría parte del Essai a la luz de las críticas de Pierre-Henri Larcher en su Supplément à la Philosophie de l'histoire (1767). ${ }^{10}$

\section{Del Essai al Esquisse: un camino regresivo}

En 1789, Condorcet escribió una Vie de Voltaire. En ella, luego de analizar la labor de Voltaire como poeta, dramaturgo y filósofo, se ocupa de su trabajo como historiador. Una vez lo ha elogiado y explicado los principales ejes de sus trabajos históricos, reconoce su deuda con el autor de Edipe. En seguida de calificar al Essai de "inmortal", expresar que "será siempre para los hombres que usan la razón una lectura deliciosa" y explicar que no es la historia de unos pocos reyes lo que interesaba al francés sino la historia del "género humano", afirma:

Es a Voltaire a quien debemos una nueva concepción de la historia más útil que la de los antiguos. Es en sus escritos que ella se ha convertido, no ya en un relato de sucesos, un cuadro de las revoluciones de un pueblo, sino de la naturaleza humana, trazado a partir de hechos, el resultado de la experiencia de todos los siglos y de todas las naciones. Es él quien ha mostrado [...] que la historia filosófica debe rechazar los hechos milagrosos [...] Estaremos siempre en deuda con Voltaire por haber eliminado de la historia esa gran cantidad de hechos extraordinarios, aceptados sin prueba alguna (Condorcet, 1847a, p. 96).

Condorcet reconoce su deuda y la de todos los historiadores modernos, entre los que destaca a los británicos Edward Gibbon, David Hume y William Robertson, con respecto a los elementos centrales de la epistemología de la historia de Voltaire, a saber, el rigor de la investigación histórica y el nuevo objeto de la historia, el género humano.

El Esquisse de Condorcet, un trabajo de los años 17931794, publicado póstumamente en 1795 y que formaba parte de un proyecto más amplio que el autor no llevó a cabo, ${ }^{11}$ parece volcarse en el molde de la filosofía de la historia de Voltaire. Condorcet -como Voltaire- reconoce desde el comienzo la importancia de apoyar el relato histórico en la experiencia y de narrar la historia del "género humano" (Condorcet, 1847b, p. 13). René Pomeau, en la introducción a su edición crítica del Essai, afirma que el Esquisse de Condorcet no es más que "una nueva versión [...] del 'plan de una historia del espíritu humano"' de Voltaire (Pomeau, 1963, p. LII.). ${ }^{12}$ Sin embargo, en el desarrollo del trabajo Condorcet se aleja poco a poco de

9. Acerca de las diferencias entre las diferentes ediciones del Essai publicadas durante el siglo XVIII, véase Pomeau, 1995, pp. 216-220.

10. Voltaire hace treinta y una modificaciones a partir del Supplément de Larcher. Entre ellas se encuentran: rectificaciones de referencias a partir de las indicaciones de Larcher (Voltaire, 1969, pp. 162, 209); adiciones de detalles tomados del Supplément (p. 212); modificaciones de datos cuestionados por Larcher (pp. 174, 209); la supresión de tesis, como la de la inexistencia de Nínive y del imperio asirio, que Larcher había refutado en su obra (p. 123).

11. El Esquisse no era más que el prospectus de una obra que llevaría por título Tableau historique des progrés de l'esprit humain y que Condorcet nunca llegó a concretar (véase Crépel, 1996). Acerca de los trabajos preparatorios de los que el Esquisse forma parte, véase el valioso de Schandeler y Crépel (Condorcet, 2004).

12. El Nouveau plan d'une histoire de l'esprit humain fue un trabajo preliminar del Essai que se publicó entre 1745 y 1746 en el Mercure de France. 
la epistemología de la historia de Voltaire, como se puede advertir al examinar la información utilizada por Condorcet y las imágenes que se desprenden de Oriente y Grecia.

\section{La historia y los hechos}

El Esquisse divide la historia en diez épocas. En las primeras páginas, Condorcet pretende seguir el desarrollo de los individuos de una generación a otra, de lo cual se desprende el objetivo del libro: presentar "el cuadro de los progresos del espíritu humano". Condorcet señala que es un "cuadro histórico" que se formará "a partir de la observación de las sociedades humanas en las diferentes épocas que han atravesado" (p. 13).

Si bien Condorcet reitera en la introducción del libro que su trabajo se apoya en la "observación" (p. 23), parece rápidamente olvidarlo. En efecto, en el primer capítulo del texto, dedicado a las primeras sociedades, explica que no hay datos sobre el asunto y que para referirse a la época se valdrá de "conjeturas" (p. 25), que se apoyan en el análisis de la naturaleza humana. Así, Condorcet se aproxima más al método de Jean Jacques Rousseau, quien en su Discours sur l'origine et les fondements de l'inégalité parmi les hommes (1755) pedía, antes de comenzar a trazar la historia de la humanidad, "dejar de lado los hechos" (Rousseau, 2008, p. 71), que al intento de Voltaire de acercar la historia a la ciencia experimental.

El modo de proceder de Condorcet no varía demasiado a medida que se acerca a épocas más cercanas a la suya, en cuanto hay en el texto una ausencia (prácticamente total) de documentos y testimonios que avalen la información vertida en las diferentes épocas (Grecia, Roma, el Medioevo, las Cruzadas, la Reforma, el siglo XVII, la Revolución americana, etc.). Se podría objetar que la situación en la que fue redactado el Esquisse (durante la redacción del trabajo, en los años 1793-1794, Condorcet se había refugiado en la casa de Mme. Vernet para escapar de una orden de arresto, elevada por la Convención, luego de su protesta contra la Constitución del 24 de junio de 1793) no le permitía un fácil acceso a los documentos. Sin embargo, la referencia a fuentes y testimonios también está ausente en los textos preliminares, como se puede observar en los manuscritos reunidos por Jean Pierre Schandeler y Pierre Crépel (Condorcet, 2004). Esos manuscritos se remontan a 1772, momento en el que Condorcet podría haber accedido a los documentos.
Por otra parte, el último capítulo del libro, dedicado a la décima época De los progresos futuros del espiritu bumano, confirma su desdén por los registros. Allí, Condorcet deja de lado la referencia al pasado para discutir acerca del futuro. Se acerca el momento, dice, en el que

El sol ya no alumbrará más que a hombres libres, que no reconocerán a otro señor que su razón, en que los tiranos o los sacerdotes y sus estúpidos o hipócritas instrumentos ya no existirán más que en la historia y en los teatros (Condorcet, 1847b, p. 244).

Condorcet anuncia con tono profético el advenimiento de un tiempo sin prejuicios, sin tiranos, sin sacerdotes. Así, mezcla una vez más, como en períodos anteriores, la historia con el relato utópico y la profecía (Baczko, 2001, p. 195). ${ }^{13}$

\section{Oriente}

En la filosofía de la historia de Condorcet, Oriente no ocupa un lugar privilegiado. Si se excluye la sexta época, las referencias a Oriente solo son marginales. A diferencia del Essai, cuyos primeros capítulos están dedicados exclusivamente a China, India, Persia y Arabia, en el Esquisse las alusiones a Oriente son poco significativas y aparecen dispersas en el libro. Oriente aparece, en todo caso, como el contrapunto de lo que Condorcet desea transmitir sobre Occidente o como una especie de terreno preparatorio respecto de Occidente (Condorcet, 1847b, p. 61).

Por otra parte, Condorcet presenta una imagen monolítica y negativa de Oriente, si bien le atribuye el origen de las artes y las ciencias. Afirma que solo en Grecia esos frutos pudieron florecer, dado que allí "las ciencias no fueron el patrimonio de una casta particular". El genio -agrega- "podía desplegar todas sus fuerzas, sin estar sometido a un control pedantesco, al sistema hipócrita de un colegio sacerdotal" (p. 61). De la misma forma, al explicar el origen de las religiones y denunciar sus objetivos, la dominación de los pueblos, afirma:

Si se quiere saber hasta qué punto pueden llevar esas instituciones su capacidad destructora de las facultades humanas hay que fijar la atención, por un momento, en China, ese lugar que parece no haber precedido a los otros en las ciencias y en las artes más que para verse sucesivamente eclipsado por todos; ese lugar en el que el conocimiento de la artillería no le ha evitado el verse conquistado por unas naciones bárbaras; en el que las ciencias, cuyas numerosas escuelas están abiertas a todos los ciudadanos [...] sin embargo, so-

13. Para una interpretación diferente, véase Waldinger, 1984. 
metidas a absurdos prejuicios, están condenadas a una eterna mediocridad; ese lugar en el que en fin la invención de la imprenta ha resultado totalmente inútil para los progresos del espíritu (p. 36).

Inmediatamente se refiere a los "vastos imperios asiáticos" como lugares donde la "ignorancia y los prejuicios" impiden los progresos y condenan el espíritu humano "a una vergonzosa inmovilidad" (p. 36). Además, al final de la sexta época, señala que el surgimiento del "genio" entre los árabes ha sido "una excepción a las leyes generales de la naturaleza que condenan a la ignorancia a las naciones sometidas y supersticiosas" (p. 123). Condorcet identifica a Oriente con el despotismo y la tiranía sacerdotal (pp. 94, 95 y 119). Estas características impiden el progreso del espíritu humano y condenan a Oriente a la ignorancia y la superstición. De esta manera y sin documentar sus afirmaciones, el pensador francés construye, como bien señala Rolando Minuti, una imagen genérica y negativa de Oriente (Minutti, 2010, p. 185).

\section{Grecia}

Voltaire se ocupa de Grecia en La Philosophie de l'histoire, trabajo que, como hemos mencionado, se convierte en 1769 en el discurso preliminar al Essai. Si bien el filósofo no deja de elogiar a los griegos por su tolerancia religiosa y de reconocer el valor de sus producciones en el campo de las artes y de la filosofía, no duda en decir que fueron "el país de las fábulas" (Voltaire, 1969, p. 173). Por otra parte, en la introducción de 1756 al Essai, lamenta -luego de recordar al lector que Occidente ha recibido las artes de Oriente- que todas las historias remitan a Grecia y Roma, naciones no solo posteriores a las de los antiguos pueblos que ocuparon Europa (galos, bretones, etc.), sino también las naciones asiáticas (Voltaire, 1963a, p. 36).

En cambio, Condorcet, hace elogio de Grecia. Salvo un comentario acerca de los abusos cometidos por los griegos en el plano de las ciencias, lo cual los llevó, dice, a "forjar sistemas" y a "entregarse a la imaginación" (Condorcet, 1847b, p. 65) alejándose de los hechos, traza en la cuarta época del libro un cuadro idílico del pueblo griego. En efecto, Grecia es presentada como el lugar donde la libertad otorgó "independencia al espíritu humano", permitiendo así el rápido progreso de las ciencias y las artes (pp. 61, 62).

Condorcet sitúa en Grecia el punto cero de la civilización. Si bien reconoce -como ya se señaló- el origen oriental de las artes, liga el conocimiento y las artes con la libertad y esa conjunción, entiende, se dio por primera vez en Grecia. Al final del capítulo dedicado a Grecia, habla de la filosofía en Grecia, de las ciencias y del alto grado que allí alcanzaron las artes. Menciona a Sófocles, a Eurípides, a Píndaro, a Tucídides, a Homero, a Platón y a Sócrates, y adelanta que se propone a partir de allí, analizar "el cuadro del progreso de estas artes, discutir sus causas [...] mostrar cómo la libertad, las artes y las luces han contribuido a la moderación y al mejoramiento de las costumbres" (p. 77). Grecia es el zócalo a partir del cual se eleva el Esquisse.

La quinta época narra el traspaso de los conocimientos griegos a Roma, la sexta, la decadencia de Roma y el advenimiento de la oscura Edad Media; los restantes capítulos, el renacimiento de las ciencias y las artes griegas, y a partir de allí, el ingreso de la historia en la segura ruta del "progreso del espíritu humano".

\section{Conclusión}

Es un lugar común considerar la filosofía de la historia de Condorcet como la continuación y consumación del proyecto historiográfico volteriano. En un trabajo célebre acerca del período, Ernst Cassirer sostuvo que "el Esquisse d'un tableau historique des progrès de l'esprit humain de Condorcet surge en derecha continuidad de las ideas y principios de Voltaire" (Cassirer, 1984, p. 245). En otro destacado libro sobre el siglo de las Luces, Jean Dagen interpreta el Esquisse como "la culminación" del proyecto volteriano y del siglo de las Luces en general (Dagen, 1977, p. 613). Asimismo, Catherine Volpilhac-Auger, en un trabajo reciente, no descarta, a pesar de poner en cuestión ciertas lecturas superficiales de la obra de Condorcet, la posibilidad de interpretar su Esquisse como "una radicalización y prolongación del combate volteriano" (Volpilhac-Auger, 2010, p. 169). Sin embargo, como hemos demostrado en estas páginas, esta interpretación corre el riesgo de pasar por alto la distancia que separa la intención de Condorcet de lo que efectivamente efectúa en el Esquisse, y por consiguiente, las tensiones entre la historia redactada por Condorcet en ese trabajo y el método historiográfico que Voltaire elaboró en los años cuarenta en Cirey y utilizó para redactar su Essai. En efecto, el Esquisse no cumple con los dos requisitos del método historiográfico de Voltaire: se aleja de los hechos hasta terminar confundiendo la historia con un relato profético, que desde el punto de vista epistemológico no se diferencia de los mitos y las fábulas, y recentraliza la historia al reconducirla hacia la tradición greco-latina. Por esta razón, podemos afirmar que antes que una consumación del proyecto historiográfico volteriano, el 
Esquisse constituye un paso atrás con respecto a la "nueva concepción de la historia” de Voltaire, de la que Condorcet se declara deudor.

\section{Referencias}

Baczko, B. (2001). Lumières de l'utopie. Paris: Payot.

Borghero, C. (1990). Les philosophes face à l'histoire: quelques discussions sur la connaissance historique aux XVIIe et XVIIIe siècles”. En Ch. Grell \& J. M. Dufays (Eds.), Pratiques et concepts de l'histoire en Europe, XVI -XVIII siècles. (pp. 73-83). París: Presses de l'Université de Paris-Sorbonne.

Brumfitt, J. (1958). Voltaire Historian. Oxford: Oxford University Press.

Cassirer, E. (1984). Filosofía de la Ilustración. México: Fondo de Cultura Económica.

Chouillet, A. M., \& Crépel, P. (1994a). Un voyage d'Italie manqué ou trois encyclopédistes réunis (D'Alembert et Condorcet chez Voltaire). Recherches sur Diderot et sur l'Encyclopédie, 17, 9-53.

Chouillet, A. M. (1994b). Le combat de Condorcet contre "l'infâme ». En T. D. Emming, et al., (Eds.). The Secular City. Studies in the Enlightenment. (pp. 170-179). Exeter: University of Exeter Press.

Chouillet, A. M. (1996). La visite de Condorcet à Voltaire et ses suites. Mélanges de l'Ecole française de Rome. Italie et Méditerranée, 108(2), 609-616.

Condorcet. (1847a). Vie de Voltaire. En M. F. Arago (Ed). Euvres complètes de Condorcet. (pp. 3-183. Vol. 4). París: Firmin-Didot.

Condorcet. (1847b). Esquisse d'un tableau historique des progrès de l'esprit humain. En F. Arago (Ed) Euvres complètes de Condorcet. (pp. 13-234. Vol. 6) Paris: Firmin-Didot.

Condorcet (2004). Tableau historique des progrés de l'esprit humain. Projets, Esquisse, Fragments, Notes. Ed. Schandeler, J. y Crépel, P. París: INED.

Cordemoy, G. (1691). Ce qu'on doit observer en écrivant l'Histoire". En Divers traitez (sic) de métaphysique, d'histoire, et de politique. París: Coignard.

Crépel, P. (1996). Esquisse d'une histoire du Tableau historique. Mélanges de l'Ecole française de Rome. Italie et Méditerranée, 108(2), 469-504.

Dagen, J. (1977). L'histoire de l'esprit humain dans la pensée française de Fontenelle à Condorcet. Paris: Klincksieck.

Grell, Ch. (1993). L'histoire entre érudition et philosophie: étude sur la connaissance historique à l'âge des Lumières. Paris: Presses universitaires de France.
Guion, B. (2008). Du bon usage de l'histoire: histoire, morale et politique à l'âge classique. Paris: Honoré Champion.

Méricam-Bourdet, M. (2012). Voltaire historien: un chantier qui s'achève?. Revue Voltaire, 12, 21-30.

Michael, C. (1987). Voltaire, Condorcet et la réhabilitation du Chevalier de La Barre. Condorcet Studies, 2, 141-154.

Minuti, R. (2010). L'Orient dans le Tableau de Condorcet: notes de lecture. En B. Binoche, (Ed.), Nouvelles lectures du Tableau historique de Condorcet. (pp. 171-199). Québec: Presses de l'Université Laval.

Pomeau, R. (2001). Voltaire et Mme du Châtelet à Cirey: amour et travail. En F. Gandt, (Ed.), Cirey dans la vie intellectuelle: La réception de Newton en France. (pp. 9-15). Oxford: Voltaire Foundation.

Pomeau, R. (1995). Voltaire en son temps. Paris: Fayard.

Pomeau, R. (1963). Introduction. Voltaire. Essai sur les mœurs et l'esprit des nations. Paris: Garnier.

Rousseau, J. J. (2008). Discurso sobre el origen y los fundamentos de la desigualdad entre los hombres. Buenos Aires: Prometeo.

Rudnik LUF, S. (2013). The Divinity of Human Making and Doing in the 18th Century. In S. Bourgault \& R. Sparling (Eds.), A Companion to Enlightenment Historiography (pp.401-436). Leiden: Brill.

Volpilhac-Auger, C. (2010). D’une histoire l'autre: Voltaire, Condorcet et l'Europe?. In B. Binoche (Ed.), Nouvelles lectures du Tableau historique de Condorcet. (pp.155-170). Québec: Presses de l'Université Laval.

Volpilhac-Auger, C. (2009). Voltaire and history. In N. Cronk (Ed.). The Cambridge Companion to Voltaire. Cambridge: Cambridge University Press.

Voltaire, (1882). Correspondance. En L. Moland (Ed.), Cuvres complètes de Voltaire. París: Garnier.

Voltaire. (1957). Nouvelles considérations sur l'histoire. París: Gallimard.

Voltaire. (1765). Histoire. Encyclopédie ou Dictionnaire raisonné des sciences, des arts et des métiers. In D. Diderot \& J. $\mathrm{D}^{\prime}$ alembert (Eds,). Encyclopédie ou Dictionnaire raisonné des sciences, des arts et des métiers (pp. 220-225. Vol. 8) París: Briasson.

Voltaire. (1963a). Essai sur les moeurs et l'esprit des nations. Ed. R. Pomeau. París: Garnier.

Voltaire. (1963b). Remarques pour servir de supplément à l'Essai sur les mours. In R. Pomeau (Ed.), Essai sur les moeurs et l'esprit des nations. (pp. 900-950). París: Garnier.

Voltaire. (1969). Philosophie de l'histoire. Oxford: The Voltaire Foundation, vol. 59

Waldinger, R. (1984). Condorcet: The Problematic Nature of Progress. Condorcet Studies, 1, 117-129. 